\title{
Assessment of rejuvenation by change of lid-cheek junction after transconjunctival and traditional subciliary incision blepharoplasty
}

\author{
Kyoung-Jin Kang, MD, PhD', Hung-Hsu Yang, MD, MS², Choong-Yee Chai, $\mathrm{MS}^{3}$ \\ ${ }^{1}$ Seoul Cosmetic Surgery Clinic, Busan, Rep. of Korea, ${ }^{2} 101$ Skin Clinic, Taipei, Taiwan, ${ }^{3}$ Korean College of Cosmetic Surgery, Busan, Rep. of Korea
}

Background: Transconjunctival lower blepharoplasty (TCLB) and traditional subciliary incision blepharoplasty (TSIB) are most commonly used to improve the appearance of an aged lower eyelid. The lid/cheek junction (LCJ) is located between the lower lid and midface, where structural changes caused by aging associated with both areas occur simultaneously. Thus, it may be a landmark that reflects the rejuvenation effects of lower blepharoplasty. However, there is no research-based proof yet.

Objective: The purpose of this study was to investigate the rejuvenation effects of these two lower blepharoplasty procedures by observing changes in LCJ length and shape.

Methods: The changes in LCJ length and shape in 32 patients with good follow-up among patients who underwent lower blepharoplasty between 2012 and 2016 were investigated. The patients were categorized as either TCLB (Group 1) or TSIB (Group 2) patients. Each group was further divided into the subgroups G1p, G1n, G2p, and G2n, according to the positive/negative globe-toskeletal relationship.

Results: The shape of LCJ changed from a V-shape to a round shape, and its length decreased in G1p, G2p, and G2n, but increased in G1n. The pattern of LCJ was also affected, corresponding to the partial deformity of the zygomaticomaxillary bone. Aged lower lids were significantly improved in all groups, but infraorbital hollowness was not improved, and indeed was even worse, in G1n.

Conclusion: Patients undergoing TCLB and TSIB surgeries significantly showed rejuvenation effects for fat protrusion, skin laxity, and wrinkles without any volume restoration, and particularly, G1n showed a worse result. Therefore, TCLB should not be recommended in $\mathrm{G} 1 \mathrm{n}$, and combination procedures that change a negative vector into a positive vector and improve infraorbital hollowness are necessary to achieve better outcomes.

Keywords: lid-cheek junction; transconjuctival lower blepharoplasty; transcutaneous lower blepharoplasty; globe-to-skeletal relationship; negative and positive vector

\section{Introduction}

When trying to improve the appearance of aged lower eyelids, understanding the simultaneous changes in anatomical structures between the lower lid and midface is key. Therefore, observing changes at the lid/cheek junction (LCJ) [1], which is located between the lower lid and midface, may be useful for classifying the degree of aging preoperatively and evaluating rejuvenation effects postoperatively.

Typically, the lid/cheek junction in young people has a gentle and round curve, and is located at or below the infraorbital rim. However, with aging, it changes to V-shape, and is always 
located below the infraorbital rim. The vertical distance from the upper edge of the lower lid to the LCJ in youthful eyes is 8-12 $\mathrm{mm}$, and this elongates to $15-18 \mathrm{~mm}$ in aged eyes in Westerners [2].

The most common surgical procedures used to improve aged lower eyelids are transconjunctival lower blepharoplasty (TCLB) and traditional subciliary incision blepharoplasty (TSIB). TCLB is the simplest method to remove protruded orbital fat by transconjunctival approach [3]. TSIB is the more traditional method of removing protruded fat and excising the lax muscle and skin by the transcutaneous approach [4], which may be combined with additional surgical procedures, such as orbital fat transposition, lateral canthopexy, suborbicularis oculi fat (SOOF) suspension suture, or midface lift, depending on the patient's preoperative conditions $[5,6]$.

In this study, the authors considered the rejuvenation effects and characteristics of these different surgeries by observing the occurrence of changes in LCJ location, according to different (positive/negative) globe-to-skeletal relationships [7].

\section{Materials and methods}

\section{Patient group}

Between 2012 and 2016, 32 patients with relatively good follow-up who underwent transconjunctival lower blepharoplasty (TCLB) and traditional subciliary incision blepharoplasty (TSIB) were chosen as study subjects. Since each patient had two eyes, the total number of cases was 62 . The patients were divided into groups as shown in Table 1.

\section{Measurement of globe-to-skeletal relationship}

The globe-to-skeletal relationship of each patient expressed as a positive or negative vector was measured as follows. Mea-

Table 1. Experimental groups

\begin{tabular}{ccccc}
\hline Group & TCLB $^{1)}$ & TSIB $^{2)}$ & $\begin{array}{c}\text { Positive } \\
\text { vector }^{3)}\end{array}$ & $\begin{array}{c}\text { Negative } \\
\text { vector }^{4)}\end{array}$ \\
\hline 1 & + & - & & \\
$1 \mathrm{p}$ & + & - & + & - \\
$1 \mathrm{n}$ & + & - & - & + \\
2 & - & + & & - \\
$2 \mathrm{p}$ & - & + & + & + \\
$2 \mathrm{n}$ & - & + & - & + \\
\hline
\end{tabular}

$\overline{{ }^{1)} \mathrm{TCLB}, \text { transconjunctival lower blepharoplasty; }{ }^{2)} \mathrm{TSIB} \text {, traditional }}$ subciliary incision blepharoplasty; ${ }^{3)}$ and ${ }^{4)}$ are globe-to-skeletal relationship. surements were taken from the lateral view photographs taken of each patient. Patients in whom the position of the cheek prominence lay beyond the anterior surface of the cornea were classified as having a positive vector. Patients in which the cheek prominence lay posterior to the surface of the cornea were classified as having a negative vector [7].

\section{Transconjunctival lower blepharoplasty (TCLB)}

TCLB was performed by modifying a method by Undavia S [5]. Two-to-three drops of topical tetracaine HCL solution were used to anesthetize the conjunctiva and cornea. An initial conjunctival incision was made using a No. 15 blade, and then electrocautery was applied for additional incisions and dissection under local anesthesia, along with a mixture of lidocaine (2\%) and epinephrine $(1: 100,000)$. The protruded fat, including that which was present underneath the orbital septum, was excised from the central and medial compartments; however, the lateral fat was not removed. Following resection of the fat, the stump was coagulated with electrocautery, and then the margin of the orbital septum was sutured with 6.0 polydioxanone monofilament for tightening of the orbital septum. After irrigation of the surgical field with $0.9 \%$ saline, the procedure was finished without conjunctival repair, to prevent irritation by suture material. Postoperatively, a 1/2-inch strip of $3 \mathrm{M}^{\mathrm{TM}}$ Micropore $^{\mathrm{TM}}$ surgical tape (3M, Maplewood, MN, USA) was placed on the lower eyelid, to support the lid and help eliminate swelling and further bleeding. Cold compression was applied for 48 hours.

\section{Traditional subciliary incision blepharoplasty (TSIB)}

TSIB was performed under local anesthesia. A skin incision was made $2-3 \mathrm{~mm}$ from the eyelash line of the lower lid. The skin flap was then dissected to the level of the junction of the preseptal and periorbital orbitalis oculi muscles using a radiofrequency module called Timed $^{\circledR}$ (TD 50A micropulse, KORPO S.R.L, Genova-Italia). The muscle incision was completed at the junction of the pretarsal-septal orbicularis muscles to preserve the whole pretarsal oculi muscle. Timed ${ }^{\circledR}$ (TD 50A micropulse, KORPO S.R.L, Genova-Italia) was also used to develop a submuscular plane between the oculi muscle and preseptal layer (complete dual plane dissection). The central and medial orbital fat, including the lax septum, were simultaneously removed, and the margin of the septum was sutured with 6.0 polydioxanone monofilament to prevent relapse of fat protrusion, and assist with further tightening of the septum. Lateral orbital fat, although protruded, was not always removed, and the lax or dehiscence septum was instead tightened or repaired. 
In G2n patients with negative vectors, SOOF suspension suture and lateral retinacular canthopexy were always performed for complication prevention. For the SOOF suspension suture, the orbital retaining ligament was divided along the inferolateral orbital rim, and the ligament, including SOOF, was resuspended superolaterally to the periosteum of the lateral orbital rim with three-point quilting sutures, made using 5-0 polydioxanone monofilament. To increase lid laxity, lateral retinacular canthopexy was performed, using the modified method of Codner MA et al. [6]. A double-armed 4-0 Prolene ${ }^{\circledR}$ (Ethicon, Somerville, NJ, USA) was used to suture the lateral retinaculum to the periosteum of the lateral orbital rim. The knot of mattress suture was placed through the periosteum within the lateral orbital rim to closely contact the posterior position of the lid margin against the globe. The muscle and skin flaps were then redraped superolaterally, and the incisions repaired by suturing with 6-0 polydioxanone mono lament and 6-0 Nylone, separately. Postoperative care was performed similarly to that following TCLB.

\section{Measurement of shape change}

\section{Measurement of lid/cheek Junction (LCJ)}

From the pre- and postoperative photographs of the patient, the LCJ was identified at six distinct points, as shown in Fig. 1, and the complete shape of the patient's LCJ was determined by connecting each of them.

\section{Measurement of bcd/ijk angle}

The angle of bcd/ijk was measured before and after the surgery using a protractor.

\section{Statistics processing method}

The length of the LCJ was measured at $\mathrm{c}$ and $\mathrm{j}$ before surgery, and at c' and j' after surgery, in patients in each group (Fig. 1). In the regularity test used by Kolmogorov-Smirnov/ShapiroWilk, although the normality of a few datasets was rejected, a parametric test was performed because its applicability was sufficient. The mean and standard deviations of each group were calculated, in the $95 \%$ confidence interval, and a paired t-test was used to carry out the pre- and postoperative comparison within the groups and subgroups. An ANCOVA test was used to compare the variations in lid/cheek junction location within the subgroups.

\section{Results}

\section{Statistical analysis}

Included were 25 women and seven men, with an average age of $42.1 \pm 11.8$ (Group 1) and 55.6 \pm 5.31 (Group 2), respectively. The follow-up period ranged from three months to two years. A statistical analysis of the changes of lid/cheek junction (LCJ) from each group was performed, and results are shown in

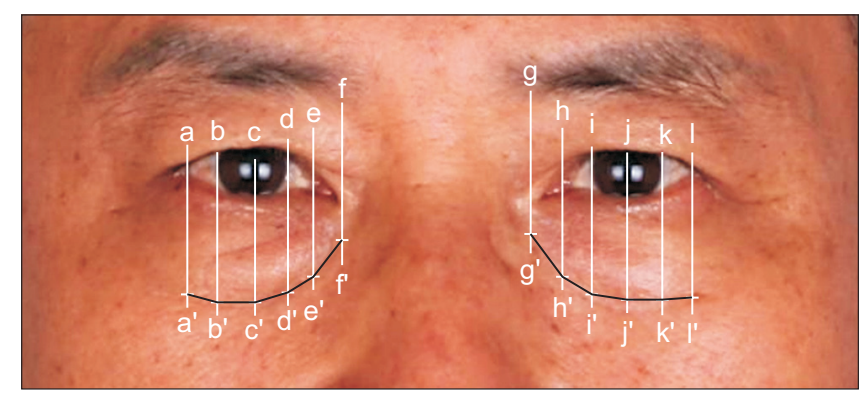

Fig. 1. Measurement of lid/cheek junction and angle of bcd/ijk. a(l)/ $a^{\prime}\left(l^{\prime}\right)$, Line drawn vertically from lateral canthus; $b(k) / b^{\prime}\left(k^{\prime}\right)$, Line drawn vertically from lateral limbus; $c(j) / c^{\prime}\left(j^{\prime}\right)$, Line drawn vertically from center of pupil; $d(i) / d^{\prime}\left(i^{\prime}\right)$, Line drawn vertically from medial limbus; $\mathrm{e}(\mathrm{h}) / \mathrm{e}^{\prime}\left(\mathrm{h}^{\prime}\right)$, The middle line in between $\mathrm{d}(\mathrm{i})$ and $\mathrm{f}(\mathrm{g})$, and $\mathrm{f}(\mathrm{g}) / \mathrm{f}^{\prime}\left(\mathrm{g}^{\prime}\right)$, Line drawn vertically from medial canthus.

Table 2. Changes and variations in the length of lid/cheek junction after TCLB and TSIB

\begin{tabular}{ccccccc}
\hline Group & $\begin{array}{c}\text { Globe-to-skeletal } \\
\text { relationship } \\
\text { (vector) }\end{array}$ & $\begin{array}{c}\text { Number of } \\
\text { cases }(\mathbf{n})^{1)}\end{array}$ & $\begin{array}{c}\text { Sex Ratio } \\
(\mathbf{M} / \mathbf{F})\end{array}$ & $\begin{array}{c}\text { Preoperative } \\
(\mathbf{m m})\end{array}$ & $\begin{array}{c}\text { Postoperative } \\
(\mathbf{m m})\end{array}$ & $\begin{array}{c}\text { Pre-post } \\
\text { Difference } \\
(\mathbf{m m})\end{array}$ \\
\hline 1 (TCLB) & - & 30 & 15 & $16.84 \pm 1.81$ & $16.70 \pm 1.99$ & $-0.14 \pm 1.55$ \\
$1 \mathrm{p}$ & Positive & 20 & $5 / 5$ & $17.13 \pm 1.91$ & $16.20 \pm 2.14$ & $-0.94 \pm 0.82$ \\
\hline $1 \mathrm{n}$ & Negative & 10 & $2 / 3$ & $16.27 \pm 1.52$ & $17.72 \pm 1.19$ & $1.45 \pm 1.45$ \\
\hline 2 (TSIB) & - & 34 & 16 & $16.99 \pm 1.94$ & $15.15 \pm 1.73$ & $-1.83 \pm 1.40$ \\
\hline $2 p$ & Positive & 24 & $3 / 9$ & $16.49 \pm 2.01$ & $14.70 \pm 1.73$ & $-1.79 \pm 1.55$ \\
\hline $2 n$ & Negative & 10 & $1 / 4$ & $18.17 \pm 1.13$ & $16.23 \pm 1.24$ & $-1.94 \pm 0.98$ \\
\hline
\end{tabular}

${ }^{1)}$ Because each patient has two eyes, the number of cases was doubled. 
Table 2.

1) In Group 1, there was no significant change of LCJ regarding length $(\mathrm{t}=0.495)$, but there was such a significant decrease in Group 2 ( $\mathrm{t}=7.655$ ) (95\% confidence interval, paired $\mathrm{t}$-test).

2) In Glp, though the length of LCJ decreased after surgery $(\mathrm{t}=5.105)$, it increased in Gln ( $\mathrm{t}=-3.14)$ (95\% confidence interval, paired t-test). Following surgery, Glp decreased significantly, compared with Gln (ANCOVA significance, $p=0.000$ ).

3) The length of LCJ decreased in both G2p and G2n ( $t=6.26$, $t=5.63$ ) (95\% confidence interval, paired t-test), but there was no difference in the LCJ length pre- and postoperatively between these subgroups (ANCOVA significance, $\mathrm{p}=0.292$ ).

\section{Morphological analysis}

Characteristic morphological changes observed from one clinical case from each group are as follows.

\section{Group $1 p$}

In this patient, the removal of protruded fat by TCLB led to the ascent of LCJ, and eventually, the length of LCJ decreased (Fig. 2C, D). The length measurements of the LCJ made at $\mathrm{c}$ and $\mathrm{j}$ both decreased. However, the length of LCJ increased at the lateral part of the right eyelid (ab->a'b'), suggesting that the LCJ moved downwards at the ab section, due to the small, flat right malar. The difference in pre- and postoperative length of LCJ at the left eye was greater than that of the right eye; this difference appears to have been caused by a more protruded left malar bone.

The angle of bcd/ijk increased on both the left and right sides, but the causal factors were different. Removal of the protruded fat near the left eyelid decreased the length of the LCJ at the c point, and led to the typical shape of a youthful LCJ, while near the right eye, this angle increased postoperatively due to the increase in length of LCJ at the ab point. This was caused by an increase of LCJ length due to a small, flat right malar bone that
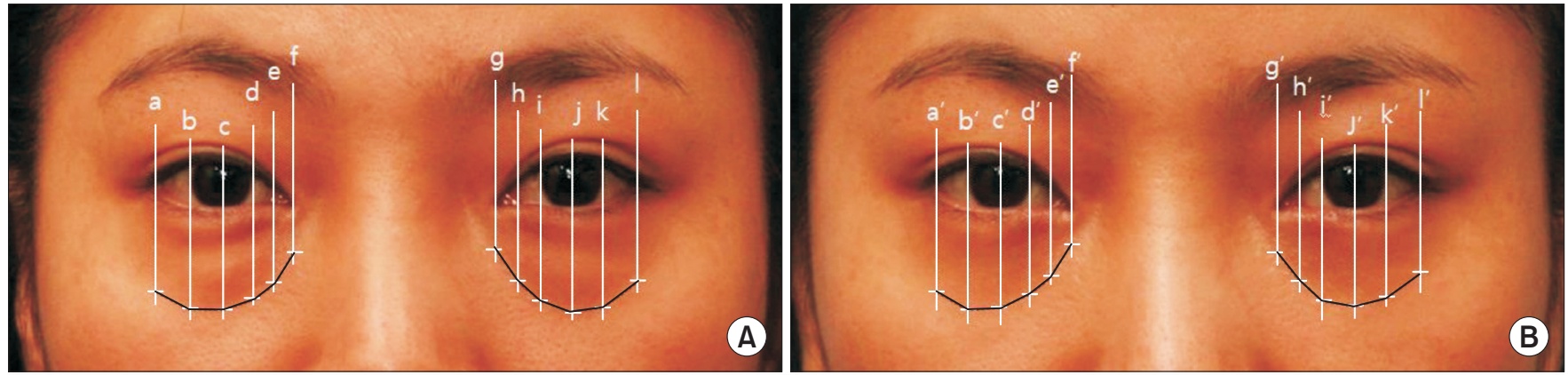

\begin{tabular}{|l|c|c|c|c|}
\hline \multirow{2}{*}{} & Bight & \multicolumn{2}{|c|}{ After (B) } \\
\cline { 2 - 5 } & 18.2 & Left & 17.3 & Left \\
\hline Length of $\mathrm{LCJ}$ in the point of $\mathrm{c}$ and $\mathrm{j}(\mathrm{mm})$ & 158 & 18.3 & 162 & 17.1 \\
\hline bcd/ijk angle $\left({ }^{\circ}\right)$ & 152 & 156 \\
\hline
\end{tabular}
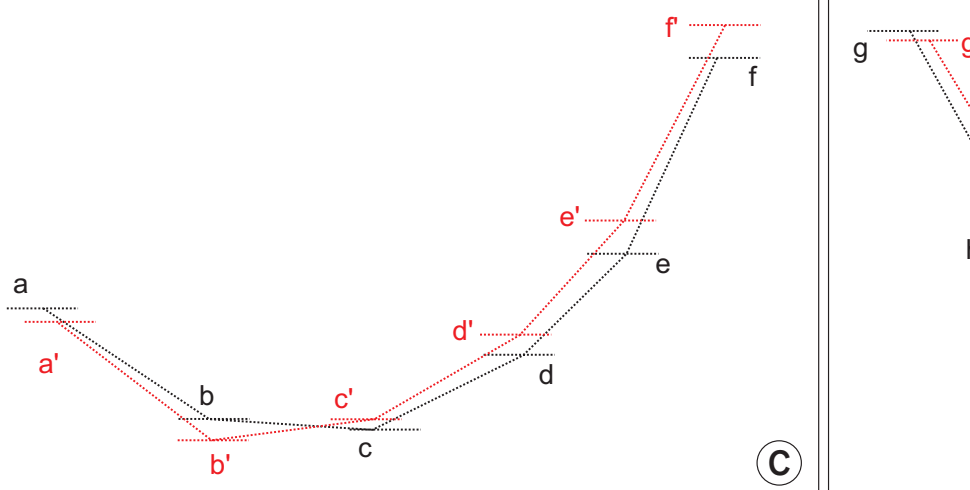

(C)

Fig. 2. A case of change of the lid/cheek junction (LCJ) and bcd/ijk angle after TCLB in a 34-year-old female patient who had a positive vector globe-to-skeletal relationship (Glp). (A) Before surgery, (B) Three months after surgery; and (C) and (D): Change of LCJ at the right (C)/left (D) eye (black dotted line means before TCLB, red dotted line means after TCLB). a-i, preoperative image; a'-i', postoperative image. 
was much greater than the decrease of LCJ length at the c point.

The protruded fat was significantly improved. However, the infraorbital hollowness seemed to have expanded and worsened.

\section{Group In}

With the removal of protruded fat by TCLB, LCJ was descended, and length was increased. It was suggested that the protruded fat was located over and in front of the infraorbital rim and was supporting the soft tissue of the lower eyelid, like the concept of a reversed negative-vector relationship. However, removal of the protruded fat caused a descent of the lower lid soft tissue. The pre-and postoperative length difference of LCJ at the left eye was greater than the right eye. This seemed to occur because the left zygomaticomaxillary bone was smaller and flatter.

The bcd/ijk angle decreased in both sides. It was suggested that the LCJ of the b-e (h-k) sections had fallen sharply, due to the removal of the protruded fats (i.e., central and inner fat), causing the angle to decrease significantly.

The protruded orbital fat was improved, but the area of infraorbital hollowness was expanded, and a sagging appearance of the lower eyelid and midface was observed.

\section{Group $2 p$}

The lengths of the identified points of the LCJ decreased overall after TSIB. However, in the medial part of the right eyelid (def->d'e'f'), the LCJ length was increased, due to a depressed naso-maxillary bone in the right side, as shown in Fig. 4A.

All bcd/ijk angles increased. This is the combined result of these points' ascension, due to the decrease in LCJ length as shown in the results of Fig. 2, and the shift in the superolateral direction of the soft tissue, due to muscle and skin redraping.

Skin laxity and protruded fat were significantly improved, but infraorbital hollowness was not improved.

\section{Group $2 n$}

All lengths of the LCJ points showed a significant decrease.
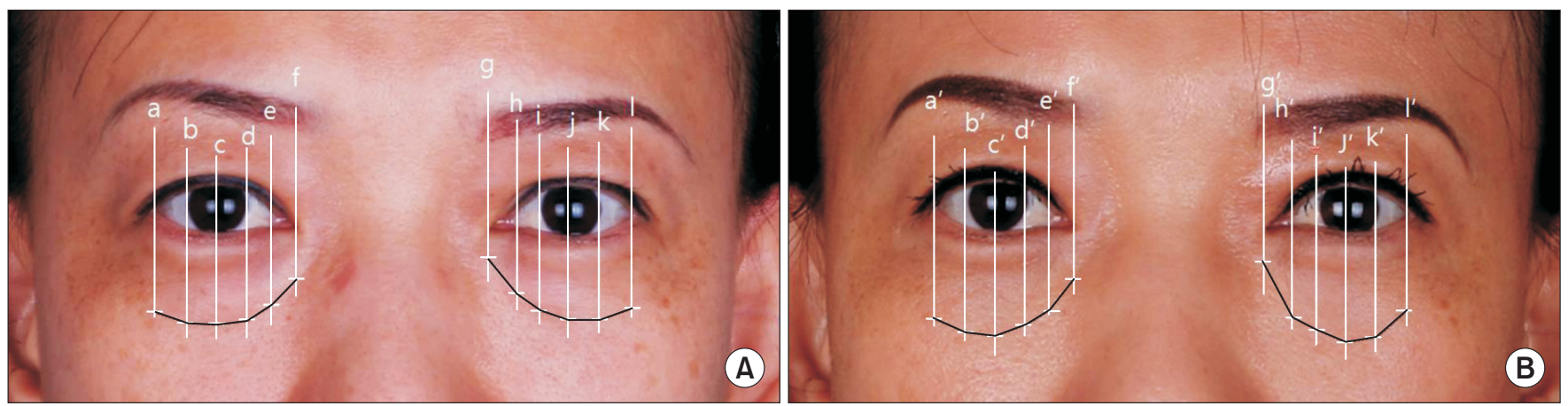

\begin{tabular}{|l|c|c|c|c|}
\hline \multirow{2}{*}{} & \multicolumn{2}{|c|}{ Before $(\mathrm{A})$} & \multicolumn{2}{|c|}{ After (B) } \\
\cline { 2 - 5 } & Right & Left & 19.5 & Left \\
\hline Length of $\mathrm{LCJ}$ in the point of $\mathrm{c}$ and $\mathrm{j}(\mathrm{mm})$ & 17 & 15.7 & 154 & 20.1 \\
\hline bcd/ijk angle ${ }^{\circ}$ ) & 168 & 160 & 152 \\
\hline
\end{tabular}
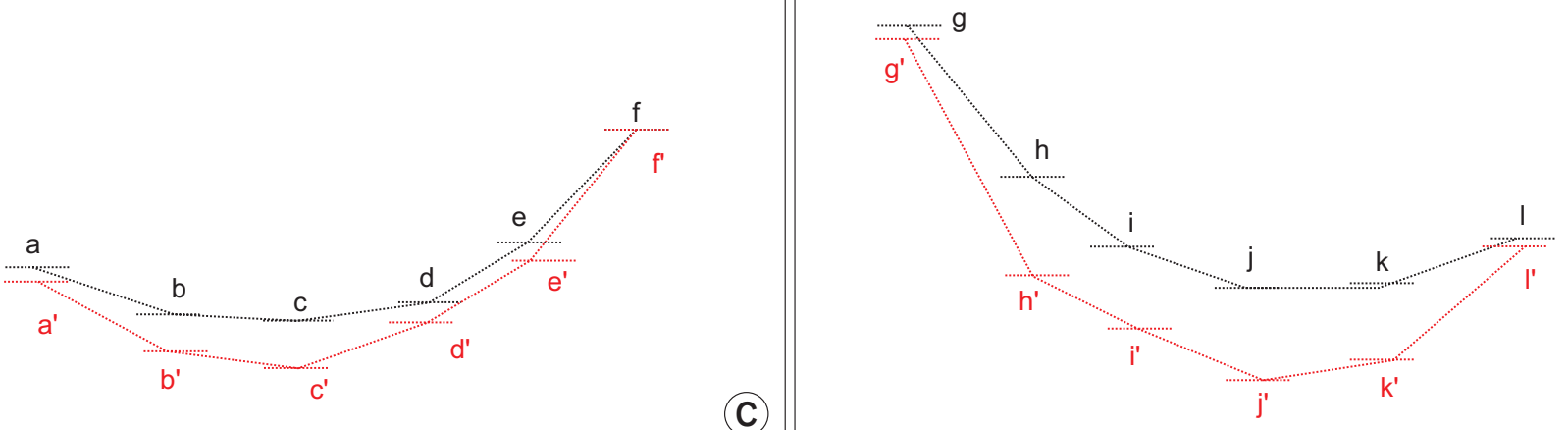

Fig. 3. A case of change of the lid/cheek junction (LCJ) and bcd/ijk angle after TCLB in a 42-year-old female patient who had a negative vector globe-to-skeletal relationship (G1n). (A) Before surgery, (B) Two years after surgery; and (C) and (D) change of LCJ at the right (C)/left (D) eye (black dotted line means before TCLB, red dotted line means after TCLB). a-i, preoperative image; a'-i', postoperative image. 

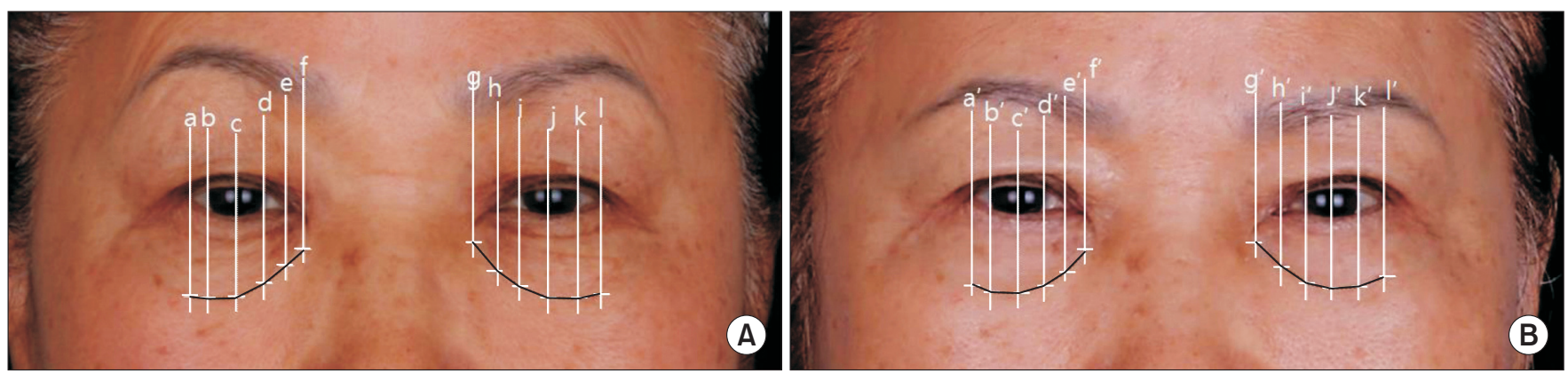

\begin{tabular}{|l|c|c|c|c|}
\hline \multirow{2}{*}{} & \multicolumn{2}{|c|}{ Before $(\mathrm{A})$} & \multicolumn{2}{|c|}{ After (B) } \\
\cline { 2 - 5 } & Right & Left & \multicolumn{2}{|c|}{ Right } \\
\hline Length of $\mathrm{LCJ}$ in the point of $\mathrm{c}$ and $\mathrm{j}(\mathrm{mm})$ & 16.3 & 16.5 & 14 & 161 \\
\hline $\mathrm{bcd} / \mathrm{ijk}$ angle $\left({ }^{\circ}\right)$ & 153 & 160 & 162 \\
\hline
\end{tabular}

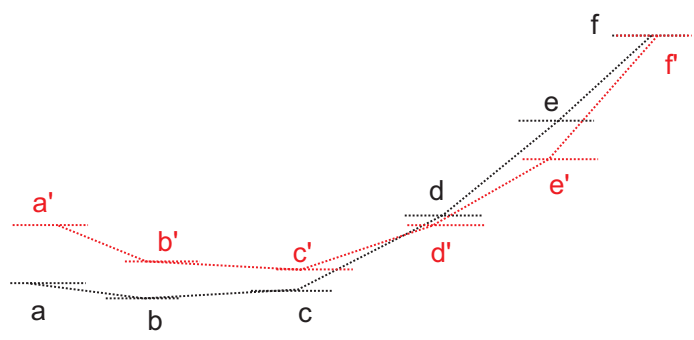

g

(C)

Fig. 4. A case of change of the lid/cheek junction (LCJ) and bcd/ijk angle after TSIB without SOOF suspension suture and lateral retinacular canthopexy in a 62-year-old female patient who had a negative vector globe-to-skeletal relationship (G2p). (A) Before surgery, (B) Six months after surgery; and (C) and (D) change of LCJ at the right (C)/left (D) eye (black dotted line means before TSIB, red dotted line means after TSIB). a-i, preoperative image; $a^{\prime}-i$,' postoperative image.

This is due to the reduced volume of protruded fat and the redraping of the skin and muscle.

All angles increased. As in Fig. 3, this occurred because the increase of the angle due to the redraping of the skin muscles was much greater than the decrease of the angle due to protruded fat removal. In this patient, the accompanied procedures of SOOF lifting and lateral canthopexy contributed significantly to the increase of the angle.

A reduction of protruded fat, skin laxity, and wrinkles was observed, but the presence of infraorbital hollowness still indicated the need for volume restoration.

\section{Discussion}

The lid/cheek junction (LCJ) is a landmark that forms the boundary between the lower eyelid and midface. It is relatively difficult to improve the signs of aging of this junctional zone because its anatomical structure is complicated, and the aging process occurs simultaneously and continuously.
This landmark is easily distinguished by differences in skin thickness between the lower eyelid and midface. On the subcutaneous plane, this is the area where the junction of the palpebral-orbicularis oculi muscle and superior border of the malar fat pad meet. On the submuscular plane, it is fixed to the orbital rim by the orbital retaining ligaments, the protruded orbital fat placed on the ligaments, and the SOOF located beneath the ligaments [8-10]. LCJ descends gradually with aging, because the vertical length of the lower lid is increased by skin and muscle laxity, protruded orbital fat, and resorption of bone [2].

TCLB and TIBS are currently the most widely and commonly used surgical methods to improve an aged lower eyelid. The rejuvenation effects of these surgeries are already well-known. Combining these with other procedures, such as fat reposition, SOOF suspension suture, lateral canthopexy, and/or midface lift for maximum results is popular $[6,11,12]$. However, currently, there is no report on changes in LCJ location induced by these two surgeries.

Hence, the authors evaluated the influence of these two sur- 
geries on the LCJ, and investigated the effects of the preoperative globe-to-skeletal relationship on the results of these two surgeries by observing the changes of LCJ.

Following TCLB, only vertical changes of the lid/cheek junction (ascend or descend) were observed; specifically, the decreased length of LCJ in G1p, and increased in G1n. In G1p with a positive vector, the soft tissues of the lower eyelid, which were pushed down by the protruded fat, ascended due to fat removal, resulting in a shorter LCJ length. This caused the angle of the middle parts (Fig. 2C-c and 2D-j) to change into a gentle curved shape. These changes indicate that the signs of aging were improved.

However, in Gln with a negative vector, the length of LCJ increased. To explain these results, the hypothesis of the authors is as follows: typically, if the orbital fat is protruded in patients with a negative vector, it is placed on and in front of the inferior orbital rim. Thus, it simulates a reversed negative vector globe- to-skeletal relationship [7] that causes the protruded fat to support the lower eyelid soft tissues. Removal of this protruded fat causes the soft tissue to descend further, increasing the length of the LCJ, and eventually turning the shape of the lid/cheek junction into a V-contour. These results showed that aging was promoted, rather than improved.

The authors' explanations are based on one study [2] proposing that LCJ descended with age. Meanwhile, in a cadaver dissection study [8], it was claimed that LCJ is unlikely to descend downward with age because of its fixation to the bone via orbital retaining ligaments. However, according to another study [13], it has been known that the orbital retaining ligaments become attenuated due to aging, and that the intra-ligamental fat of the retaining ligaments also decreases. The authors showed the results of the decreased length of the LCJ without releasing the orbital retaining ligaments from the orbital rim during TSIB (Fig. 4). This means that the LCJ is not a fixed structure, but a
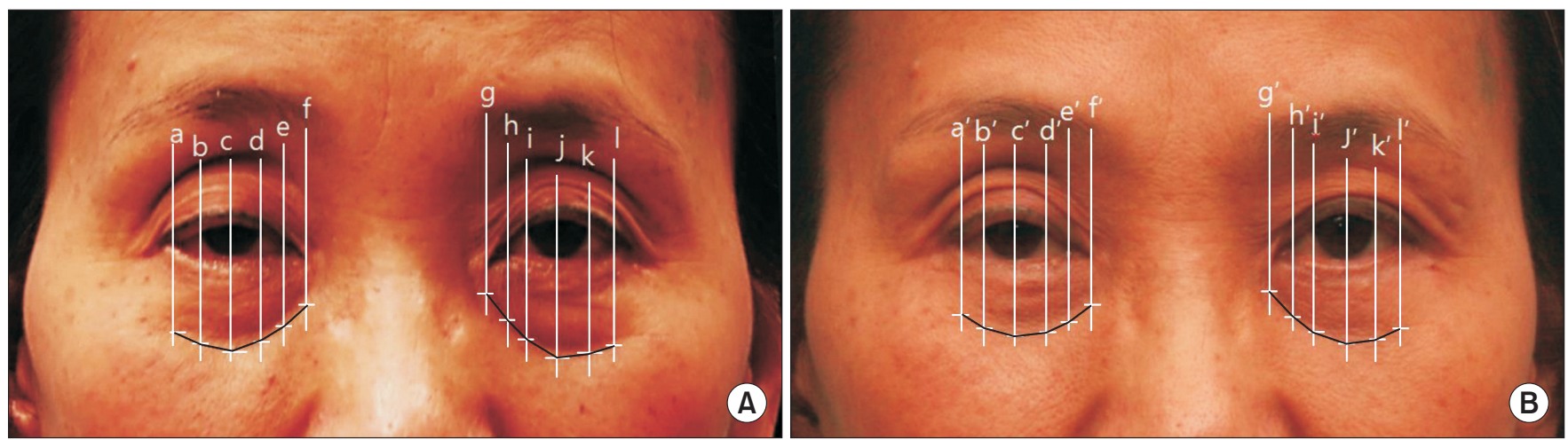

\begin{tabular}{|l|c|c|c|c|}
\hline \multirow{2}{*}{} & \multicolumn{2}{|c|}{ Before $(\mathrm{A})$} & \multicolumn{2}{|c|}{ After (B) } \\
\cline { 2 - 5 } & Right & Left & 13.5 & Left \\
\hline Length of $\mathrm{LCJ}$ in the point of $\mathrm{c}$ and $\mathrm{j}(\mathrm{mm})$ & 17.3 & 17.0 & 162 & 15 \\
\hline bcd/ijk angle $\left({ }^{\circ}\right)$ & 149 & 142 & 153 \\
\hline
\end{tabular}

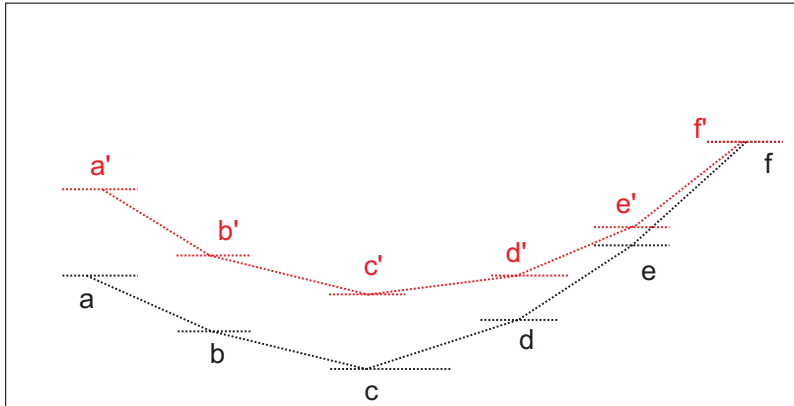

(C)

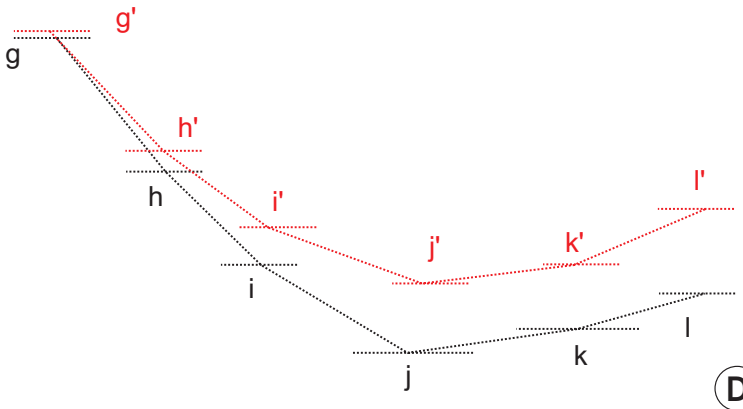

Fig. 5. A case of change of the lid/cheek junction (LCJ) and bcd/ijk angle after TSIB with SOOF suspension suture and lateral retinacular canthopexy in a 55-year-old female patient who had a negative vector globe-to-skeletal relationship (G2p). (A) Before surgery, (B) three months after surgery; and (C) and (D) change of LCJ at the right (C)/left (D) eye (black dotted line means before TSIB, red dotted line means after TSIB). a-i, preoperative image; a'-i', postoperative image. 
movable structure whose position depends on changes in the surrounding environment.

In Group 2, after TIBS, the length of LCJ was decreased, regardless of the influence of the globe-to-skeletal relationship. The V-shape of the LCJ was changed to a gentle round shape, which was a smoother appearance than that seen in Group 1, caused by vertical and horizontal movements of the LCJ after TIBS. This was the corresponding result from the methodological differences between the TCLB and TIBS.

Although there was no difference in length of LCJ between G2p and G2n according to statistical analysis, a patient with a negative vector (Fig. 5) demonstrated greater variation of LCJ location than a patient with a positive vector (Fig. 4); this was likely due to the application of different surgical procedures. However, in fact, almost all the negative vector patients and a few of the positive vector patients underwent a combination of TCLB or TIBS and additional procedures such as SOOF suspension suture, lateral retinacular canthopexy, and/or subperiosteal midface lift. This is the main reason for no statistical difference in the length of LCJ after TIBS. In general, the preand postoperative differences of LCJ location in patients who underwent additional procedures was greater than that of the patients who underwent only TIBS.

Preoperatively, the globe-to-skeletal relationship was measured from the lateral view of the face following the conventional method [7]; that is, when looking from the front, the measurements were obtained from the middle of the LCJ (position of Fig. 1c and 1j). Fig. 2C and Fig. 4C showed that partial deformities of the zygomaticomaxillary bone, such as flatness or depression, caused partial changes in the length and shape of LCJ, which resulted in a more sagged appearance or further expanded infraorbital hollowness. These results suggest that partial deformities of the zygomaticomaxillary bones should be considered as distinct factors that affect the results of lower blepharoplasty procedures.

In this study, TCLB showed an improvement in protruded orbital fat, and TIBS showed significant improvements in protruded orbital fat, skin laxity, and wrinkles; however, no significant improvement on infraorbital hollowness was observed in either of the two surgeries. Infraorbital hollowness consists of tear trough deformity [8], mid/cheek depression, and palpebromalar groove [14]. It is caused by the combination of bone resorption with aging, descent and volume reduction of fat, and laxity of the soft tissues. Hence, performing TSIB alone cannot bring maximum results: it is essential to additionally perform volume restoration procedures, such as fat reposition, free fat graft, filler injection, and implantation, simultaneously.

In addition, the infraorbital hollowness in Gln patients with a negative vector was more expanded and further sagged following TCLB. It is strongly suggested that patients with negative vectors should be simultaneously treated by volume restoration surgery, which changes the negative vector into a positive vector, together with TCLB.

\section{Conclusion}

This study indicates that the changes of LCJ due to TCLB and TSIB procedures, as an indicator of rejuvenation of aged lower lid, are proven to be useful. TCLB and TSIB surgical methods also showed distinct characteristic morphological changes of LCJ, differing according to each method (TCLB: vertical change; TSIB: vertical and horizontal change). Surgeons should note that a single treatment of TCLB should not be recommended to patients with negative vectors. Lastly, in some cases, TCLB and TSIB could not completely rejuvenate and/or might have worsened the aged lower lid. This was because the procedures only had the effects of reducing volume and improving sagging, without any accompanying volume restoration.

\section{Conflicts of interest}

The authors have nothing to disclose.

\section{References}

1. Lambros V. Observations on periorbital and midface aging. Plast Reconstr Surg 2007;120:1367-76; discussion 1377.

2. Hester TR Jr, Codner MA, McCord CD, Nahai F, Giannopoulos A. Evolution of technique of the direct transblepharoplasty approach for the correction of lower lid and midfacial aging: maximizing results and minimizing complications in a 5-year experience. Plast Reconstr Surg 2000;105:393-406; discussion 407-8.

3. Tomlinson FB, Hovey LM. Transconjunctival lower lid blepharoplasty for removal of fat. Plast Reconstr Surg 1975;56:314-8.

4. Zarem HA, Resnick JI. Expanded applications for transconjunctival lower lid blepharoplasty. Plast Reconstr Surg 1991;88:215-20; discussion 221.

5. Undavia S, Briceño CA, Massry GG. Quantified incision placement for postseptal approach transconjunctival blepharoplasty. Ophthal Plast Reconstr Surg 2016;32:191-4.

6. Codner MA, Wolfli JN, Anzarut A. Primary transcutane- 
ous lower blepharoplasty with routine lateral canthal support: a comprehensive 10-year review. Plast Reconstr Surg 2008;121:241-50.

7. Yaremchuk MJ. Restoring palpebral fissure shape after previous lower blepharoplasty. Plast Reconstr Surg. 2003;111:44150.

8. Haddock NT, Saadeh PB, Boutros S, Thorne CH. The tear trough and lid/cheek junction: anatomy and implications for surgical correction. Plast Reconstr Surg 2009;123:1332-40; discussion 1341-2.

9. Segal KL, Patel P, Levine B, Lisman RD, Lelli GJ Jr. The effect of transconjunctival blepharoplasty on margin reflex distance 2. Aesthetic Plast Surg 2016;40:13-8.

10. Palmer FR 3rd, Rice DH, Churukian MM. Transconjunctival blepharoplasty. Complications and their avoidance: a retro- spective analysis and review of the literature. Arch Otolaryngol Head Neck Surg 1993;119:993-9.

11. Goldberg RA. Transconjunctival orbital fat repositioning: transposition of orbital fat pedicles into a subperiosteal pocket. Plast Reconstr Surg 2000;105:743-8; discussion 749-51.

12. Turk JB, Goldman A. SOOF lift and lateral retinacular canthoplasty. Facial Plast Surg 2001;17:37-48.

13. Kikkawa DO, Lemke BN, Dortzbach RK. Relations of the superficial musculoaponeurotic system to the orbit and characterization of the orbitomalar ligament. Ophthal Plast Reconstr Surg 1996;12:77-88.

14. El-Garem YF. Estimation of bony orbit depth for optimal selection of the injection technique to correct the tear trough and palpebromalar groove. Dermatol Surg 2015;41:94-101. 